\title{
Area-Type Adjustment Factors for Non-CBD Signalised Intersections
}

\author{
Abena Agyeiwaa Obiri-Yeboah ${ }^{1}$, Yaw Adubofour Tuffour ${ }^{2,}$, Mohammed Salifu $^{2}$ \\ ${ }^{1}$ Department of Civil Engineering, Kumasi Polytechnic, Kumasi, Ghana \\ ${ }^{2}$ Department of Civil Engineering, Kwame Nkrumah University of Science and Technology, Kumasi, Ghana
}

Email address:

aanyarko@yahoo.co.uk (A. A. Obiri-Yeboah), yat@engineer.com (Y. A. Tuffour), salifum@hotmail.com (M. Salifu)

*Corresponding author

\section{To cite this article:}

Abena Agyeiwaa Obiri-Yeboah, Yaw Adubofour Tuffour, Mohammed Salifu. Area-Type Adjustment Factors for Non-CBD Signalised Intersections. American Journal of Civil Engineering. Vol. 5, No. 2, 2017, pp. 119-123. doi: 10.11648/j.ajce.20170502.17

Received: October 28, 2016; Accepted: January 4, 2017; Published: March 4, 2017

\begin{abstract}
In the estimation of saturation flow at signalized intersections using the HCM model, the influence of area-type characteristics on flow is accounted for by the area-type adjustment factor $\left(f_{a}\right)$. The recommendation by the manual to use $f_{a}=0.9$ for CBD-intersections and 1.0 for all others tacitly assumes that the nature of non-CBD areas does not influence flow. This may not hold for many developing country cities and metropolitan areas where the roadside environments associated with non-CBD intersections may be laden with a flurry of activities that impact traffic flow. For signalized intersections in such areas, the HCM-recommended area-type adjustment factor could lead to over-estimation of saturation flow rates if the activities create substantial roadside friction to flow. This study set out to develop area-type adjustment factors for non-CBD signalized intersections located within the Kumasi Metropolis in Ghana, to accurately reflect the impact of roadside character on saturation flow rates. Using field saturation flow data collected at selected signalized intersections in the metropolis, area-type adjustment factors were developed for three distinct roadside friction environments categorised in the study as low/none, medium, and high. Average $f_{a}$ values developed were 0.99 for low/none, 0.98 for medium, and 0.94 for high roadside friction environments. Also, a multiple linear regression model that attempts to relate $f_{a}$ to pedestrian traffic interfering with flow within the environment of the intersection was developed as an alternative procedure for determining site specific area-type adjustment factor. It is recommended to use the $f_{a}$ values developed in this study in the HCM saturation flow model in order to estimate more accurately the saturation flow rates at non-CBD signalized intersections within the Kumasi Metropolis.
\end{abstract}

Keywords: Area-Type Adjustment Factor, Non-CBD Signalised Intersections, Saturation Flow, Roadside Friction

\section{Introduction}

Direct field measurement of saturation flow rates associated with vehicular discharge through signalized intersections forms part of the routine for assessing the performance of such intersections. The alternative procedure to field measurements recommended by the Highway Capacity Manual (HCM) [1] is to adjust the ideal flow of 1900pcphgpl by a number of factors to reflect all the parameters that cause the flow to deviate from ideal. The manual provides empirical expressions for evaluating the adjustment factors based on parameters associated with the traffic stream, geometrics, and external interference at the intersection. Due to variability in local conditions, the adjustment factors recommended by the manual have become a subject of interest to researchers around the world in the effort to adapt the manual to suit local conditions.

Shao et al. [2] studied the influence of traffic composition, lane widths, and approach grades on signalised intersections in China and developed factors that were inconsistent with corresponding factors in the HCM [1]. The adjustment factors were developed for different combinations of lane widths and turning radii reflective of the local roadway geometrics. Rahman et al. [3] derived adjustment factors based on data collected from Yokohama City, Japan, and noted that saturation flow rates increased by $20 \%$ which corresponded to a professional driver adjustment factor of 1.20 if the signalised intersection was identified to have a high volume of taxi drivers. 
External interference to signalised intersection flow from the activities taking place in the immediate environment of the intersection constitutes side friction to flow. Chiguma [4] has noted that side friction may result from interference to flow on the travelled lanes or blockage of the travelled way by public transport vehicles using passenger facilities located close to the intersection, pedestrians, and street-side hawking among others. The HCM model uses the area-type adjustment factor $\left(f_{a}\right)$ to account for the impact on saturation flow of the area within which a signalised intersection is located. Two area type categories are recognized by the model; CBD, and all others. Hence, the manual recommends the use of $f_{a}=0.9$ for CBD intersections and 1.0 for all others. The lower factor is in recognition of the reduction in efficiency of intersections in CBDs due to friction from high pedestrian-related activities, vehicle-pedestrian interactions, reduced street widths from on-street parking, and frequent parking manouevres close to such locations.

In the case of non-CBDs, the assumption implicit in the use of $f_{a}=1.0$ is that the intensity of activities characterizing such areas is low and, hence, vehicular discharge is not affected by the level of activities. This recommendation appears too over-generalized since non-CBD locations in many countries can have diverse characteristics and may be laden with activities with different degrees of intensity which may impact flow differently. Le et al. [5] have rightly noted that the types of areas associated with non-CBDs vary and may include business, recreational, shopping, and residential. Based on the above area types, a factor of 0.92 was established for recreational areas and 1.0 for all others [5]. In more general terms, Bonneson et al. [6] have noted that the amount of the reported decrease in terms of adjustment factor for flow at non-CBD intersections may vary from 0.90 to 0.99 . This, therefore, suggests that placing intersection area types into only two categories for the purposes of flow estimation may not suffice in all situations.

\section{Related Works}

The need to develop area-type adjustment factors for signalized intersections in Ghanaian cities is even more imperative and relevant considering the fact that most roadside areas close to such intersections are associated with a lot of pedestrian and vehicular activities [7]. In more general terms, the effect of roadside pedestrian interference to traffic flow may manifest in three different ways; 1) increase in the start-up delay when drivers in front of the queue delay further to allow pedestrians who are crossing at the onset of vehicular green to complete their crossing manoeuvre; 2) delay caused to drivers upstream when on-street pedestrian hawkers continue to engage the attention of drivers and other vehicle occupants in the discharging queue and prevent them from responding quickly to the release shock wave following the onset of green; and 3) slowdown of approaching vehicles when some pedestrians use or attempt to use large gaps in staggered flows to cross the road [7].
Whereas the impacts of some elements of roadside friction to flow, such as the blocking effect of stopping and parking vehicles, are dealt with by specific factors in the HCM saturation flow model, those of pedestrian interference and friction elements not readily apparent are not and may be taken as collectively embodied in the area-type factor. Therefore, conceptually, the area-type adjustment factor may be seen as adjusting for the effects of friction elements within the roadside environment which cannot individually be assigned specific adjustment factor values. This concept may be seen as re-echoing the recognition by Tarko and Tracz [8] that omission of some capacity factors in predictive models and inadequate functional relationship between model variables and saturation flow rates introduce certain level of errors for existing capacity analysis. The present study, which was an extension of previous studies $[9,10]$ on signalized intersection flows within the Kumasi Metropolis, was aimed at developing area-type adjustment factors that reflect local conditions when the HCM model is to be used as the tool for the estimation of saturation flows.

\section{Development of Area-Type Adjustment Factors}

The HCM [1] model for computing saturation flow rates is provided as;

$$
S=S_{o} n f_{w} f_{H V} f_{g} f_{p} f_{b b} f_{a} f_{L U} f_{R T} f_{L T} f_{L p b} f_{R p b}
$$

where,

$\mathrm{S}=$ saturation flow rate for the lane group in passenger car per hour of green.

$\mathrm{S}_{\mathrm{o}}=$ ideal saturation flow rate in pcphgpl

$\mathrm{n}=$ number of lanes in the lane group

$\mathrm{f}_{\mathrm{w}}=$ adjustment factor for lane width

$\mathrm{f}_{\mathrm{HV}}=$ adjustment factor for heavy vehicles

$f_{g}=$ adjustment factor for approach grade

$\mathrm{f}_{\mathrm{p}}=$ adjustment factor for parking characteristics

$\mathrm{f}_{\mathrm{bb}}=$ adjustment factor for blocking effect of local buses that halt within the intersection area

$\mathrm{f}_{\mathrm{a}}=$ adjustment factor for area type

$\mathrm{f}_{\mathrm{LU}}=$ adjustment factor for lane utilization

$\mathrm{f}_{\mathrm{RT}}=$ adjustment factors for right-turns in the lane group

$\mathrm{f}_{\mathrm{LT}}=$ adjustment factors for left-turns in the lane group

$\mathrm{f}_{\mathrm{Lpb}}=$ pedestrian-bicycle adjustment factor for left-turn movements; and

$\mathrm{f}_{\mathrm{Rpb}}=$ pedestrian-bicycle adjustment factor for right-turn movements.

To account for the friction effect due to bus blockage at signalized intersections within the Kumasi Metropolis, and to reflect more accurately the character of local public transport vehicles and driver behavior in the metropolis, Tuffour et al. [10] suggested modifications to the empirical expression for the bus blockage factor in the HCM model as follows: 


$$
f_{b b}=1-\frac{t_{b}\left(N_{\text {buses }}+\frac{P C E_{\text {taxi }}}{P C E_{\text {bus }}} N_{\text {taxi }}+\frac{P C E_{\text {tro }}}{P C E_{\text {bus }}} N_{\text {tro }}\right)}{3600 \mathrm{~N}}
$$

where,

$\mathrm{t}_{\mathrm{b}}=$ bus blockage time

$\mathrm{N}_{\text {buses }}=$ number of buses

$\mathrm{N}_{\text {taxi }}=$ number of taxis

$\mathrm{N}_{\text {tro }}=$ number of trotros

$\mathrm{PCE}_{\mathrm{bus}}=$ passenger car equivalent of bus

$\mathrm{PCE}_{\text {taxi }}=$ passenger car equivalent of taxi

$\mathrm{PCE}_{\text {tro }}=$ passenger car equivalent of trotro

The modification was also to recognize the fact that in Ghana, intra-city public transportation services are offered using predominantly minibuses which are locally referred to as trotros. Conventional large capacity public transport vehicles/buses are used mostly for long-distance inter-city travels. In addition, taxis operate almost exclusively in shared-passenger mode and behave much in the same way as the trotros. Therefore, at any signalised intersection, the public transport vehicles involved in blockage to intersection discharge flow would be mainly trotros and taxis and a limited number of conventional buses. Adopting the modification suggested by Eq. (2) and using a composite factor $F$ to represent all adjustment factors other than the area-type factor, Eq. (1) may be re-written as;

$$
S=n F f_{a} S_{o}
$$

If the saturation flow estimated from the HCM ideal flow model must match the field saturation flow, then

$$
f_{a}=\frac{S_{\text {field }}}{n F S_{o}}
$$

where,

$\mathrm{S}_{\text {field }}=$ field (observed) saturation flow rate

$\mathrm{F}=$ composite adjustment factor for all factors (excluding the area-type factor).

Equation (4) formed the basis for developing new area-type adjustment factors appropriate to the roadside conditions associated with non-CBD signalised intersections within the Kumasi Metropolis.

\section{Methodology}

\subsection{Categorization of Signalized Intersections}

A total of 11 signalized intersections, all controlled by pre-timed signals, were selected within the Kumasi Metropolis for the study. The intersections were placed in three roadside friction categories, namely, low/none, medium, and high friction intersections according to the guide in Table 1 suggested by Tuffour et al. [10] for signalized intersections within the metropolis. It is recognized that the categorization is more subjective than objective but nevertheless provides a coarse basis for distinguishing between the different intersection area friction regimes.

Table 1. Guide for categorizing roadside friction at signalised intersections [10].

\begin{tabular}{lll}
\hline \multicolumn{2}{l}{ Roadside Friction Categories } & \\
\hline Low/None & Medium & High \\
\hline At most one of these & Two of the following & At least three of the \\
friction agents must & friction agents must & following friction \\
prevail: & prevail: & agents must prevail: \\
Taxi Rank & Taxi Rank & Taxi Rank \\
Lay-by/Bus Bay & Lay-by/Bus Bay & Lay-by/Bus Bay \\
Fuel Station & Fuel Station & Fuel Station \\
Trotro Station & Trotro Station & Trotro Station \\
& &
\end{tabular}

\subsection{Data Collection}

Real time traffic movements through the signalised intersections for a two-hour duration during the morning peak flow were recorded over several cycles using a portable digital camcorder mounted on a tripod. Video recording was used to provide accurate records of traffic flow and also make it possible, when necessary, to review the impact of special conditions through playback at the data extraction stage. Vehicular headways extracted from the recordings using the playback method on a computer screen were converted to saturation flow rates using the expression;

$$
S_{f_{\text {ield }}}=\frac{3600}{h}
$$

where, $S_{\text {field }}=$ field (observed) saturation flow rate and $h$ is the average saturation headway in seconds.

Data associated with the gradients and geometrics at the intersections and other parameters required for computing all the relevant adjustment factors in the HCM model were collected manually. Passenger car equivalent values developed by Obiri-Yeboah et al. [9] for signalized intersections within the metropolis (see Table 2) were used in computing the modified bus blockage factor suggested by Tuffour et al. [10]. The bus blockage time $\left(t_{b}\right)$ in Eq. (2) was selected to reflect appropriate public transport driver behaviour in each of the intersection types per the values recommended by Tuffour et al. [10].

Table 2. PCE values for different vehicle categories [9].

\begin{tabular}{lll}
\hline \multirow{2}{*}{ Vehicle Category } & \multicolumn{2}{l}{ Intersection Type } \\
\cline { 2 - 3 } & With Roadside Friction & Without Roadside Friction \\
\hline Cars & 1.00 & 1.00 \\
Medium Vehicles & 1.65 & 1.35 \\
Trucks & 3.05 & 2.25 \\
\hline
\end{tabular}

\section{Results and Discussion}

Table 3 is a summary of the signalized intersection types by friction categories considered in the study, the field saturation flows, adjusted saturation flows, and the corresponding area-type factors developed. It is to be noted that the intersection names used in the table reflect those used by residents of the metropolis for identification rather than the names of the intersecting roads. Based on the $S_{\text {field }}$ values, the 
average flow rates (in pcphgpl) associated with each of the intersection friction categories were 1250 for high, 1405 for medium and 1680 for low/none. Clearly, the averages provide levels of differentiation between the intersection area types which appear to be in tandem with the subjective friction categorization adopted in this study. For the metropolis, Obiri-Yeboah et al. [9] had observed that saturation headways were generally lower at low/none friction intersections than at medium and high friction ones as expected. Lower headways translate to higher saturation flow rates for the low/none friction intersections.

In the case of the area-type adjustment factors for the different friction environments, the average $f_{a}$ values developed were; 0.99 for the low/none friction areas, 0.98 for the medium friction areas and 0.94 for high friction areas (see Table 3). The range of $f_{a}$ values obtained in this study is in sync with that of 0.9-0.99 suggested by Bonneson et al. [6] for non-CBD intersections. It also indirectly vindicates the concerns expressed by Tarko and Tracz [8] that there are shortcomings in predictive models in capacity analysis due to inadequate functional relationship between model variables and saturation flow rates.

For the intersection environments categorised as low/none friction area, $f_{a}=0.99$ obtained in this study is in very good agreement with $f_{a}=1.00$ recommended by the HCM. On the other hand, the value of $f_{a}=0.94$ derived for areas considered to impose high friction on flow is substantially lower than $f_{a}=1.00$, implying that the recommended HCM value is inappropriate for such area types within the metropolis as it would lead to overestimates of flows.

As explained early on, most of the friction agents and accompanying activities generate pedestrian traffic that tends to interfere with traffic flow. In view of this, an attempt was made to relate the hourly volumes of pedestrian traffic interfering with traffic flow at the intersections to the area-type adjustment factors. This led to the following multiple linear relationship with a coefficient of determination, $R^{2}=0.93$.

$$
\mathrm{f}_{\mathrm{a}}=0.9945-6.9 \times 10^{-5} \mathrm{~N}_{\mathrm{pc}}-0.00039 \mathrm{~N}_{\mathrm{ph}}, \mathrm{R}^{2}=0.93
$$

where,

$f_{a}=$ area-type adjustment factor

$N_{p c}=$ number of crossing pedestrians

$N_{p h}=$ number of pedestrian hawkers

As is obvious from the above relationship, where friction to flow from pedestrian interferences do not exist, a value of $f_{a}=0.9945$, which corresponds essentially to the low/none intersection friction category, would be returned. This approach to area-type adjustment factor development in this study is yet to be extended to other metropolitan areas where roadside friction to intersection flow exists.

Table 3. Summary of area-type adjustment factors.

\begin{tabular}{|c|c|c|c|c|c|c|}
\hline Intersection Name & Approach & Roadside Friction Category & $\mathrm{nS}_{0} \mathrm{~F}$ (pcphgpl) & $S_{\text {field }}$ (pcphgpl) & $f_{a}$ & Average $f_{a}$ \\
\hline \multirow{2}{*}{ Brigina } & Stadium & \multirow{6}{*}{ Low/None } & 1740 & 1640 & 0.94 & \multirow{6}{*}{0.99} \\
\hline & Asafo & & 1620 & 1650 & 1.02 & \\
\hline \multirow{2}{*}{ Pine Avenue } & Adum & & 1740 & 1730 & 0.99 & \\
\hline & Officers Mess & & 1710 & 1730 & 1.01 & \\
\hline \multirow{2}{*}{ Stadium } & Childrens' Park & & 1710 & 1710 & 1.00 & \\
\hline & Asokwa & & 1640 & 1610 & 0.98 & \\
\hline Bomso & University & \multirow{5}{*}{ Medium } & 1330 & 1320 & 1.00 & \multirow{5}{*}{0.98} \\
\hline \multirow{2}{*}{ Amakom } & Afful Nkwanta & & 1570 & 1550 & 0.99 & \\
\hline & Labour & & 1560 & 1530 & 0.98 & \\
\hline \multirow{2}{*}{ Asokwa } & Asafo & & 1250 & 1220 & 0.97 & \\
\hline & Atonsu & & 1450 & 1440 & 1.00 & \\
\hline \multirow{2}{*}{ Kurofrom } & Dichemso & \multirow{9}{*}{ High } & 1450 & 1360 & 0.93 & \multirow{9}{*}{0.94} \\
\hline & Suame & & 1460 & 1329 & 0.92 & \\
\hline \multirow{2}{*}{ Aboabo } & Anloga & & 1290 & 1290 & 1.00 & \\
\hline & Dichemso & & 1270 & 1240 & 0.98 & \\
\hline Abrepo Junction & Neoplan & & 1320 & 1190 & 0.90 & \\
\hline \multirow{2}{*}{ Anloga Junction } & Oforikrom & & 1340 & 1290 & 0.96 & \\
\hline & Afful Nkwanta & & 1310 & 1170 & 0.89 & \\
\hline \multirow{2}{*}{ University Crossing } & Top High & & 1360 & 1260 & 0.93 & \\
\hline & Kentinkrono & & 1290 & 1210 & 0.93 & \\
\hline
\end{tabular}

\section{Conclusion}

The paper has presented the procedures involved in the development of area-type adjustment factors for saturation flow estimation for use in non-CBD areas within the Kumasi Metropolis. The study was motivated by the need to develop area-type adjustment factors that reflected the true nature of the varied intersection environments in non-CBD areas within the metropolis. The recommendation by the HCM to use $f_{a}=0.9$ for $\mathrm{CBD}$ intersections and $f_{a}=1.00$ for all other intersections indirectly but erroneously assumes that the nature of non-CBD intersections areas does not influence intersection discharge. Where there is significant intersection area interference, the recommendation could lead to overestimation of flow. The area-type adjustment factors developed placed the intersection environment in three friction categories, namely, low/none, medium, and high 
friction areas. Area-type adjustment factors were developed for the three categories of intersections using saturation flow rates measured in the field and the HCM predictive model with passenger car equivalent values developed in earlier studies for the intersections as part of the input parameters. Average $f_{a}$ values obtained were 0.99 for low/none, 0.98 for medium, and 0.94 for high friction environments. A multiple linear regression model that attempted to relate $f_{a}$ to pedestrian traffic interfering with vehicular flow at the intersections was developed as an alternative procedure for determining site specific area-type adjustment factors. It is recommended that area-type adjustment factors developed in this study be used in the HCM estimation model for predicting the saturation flow rates at signalized intersections within the Kumasi Metropolis to reflect accurately the effect of local traffic and roadside environment conditions on flow.

\section{References}

[1] Highway Capacity Manual (2010). Transportation Research Board, National Research Council, Washington, DC.

[2] Shao, C. and Liu, X. M. (2011). "Study on the Saturation Flow Rate and Its Influence Factors at Signalised Intersections in China", Procedia Social and Behavioral Sciences, 16 (2011) 504-514.

[3] Rahman, M. and Nakamura, F. (2005). "Measuring passenger car equivalents for non-motorized vehicle (Rickshaws) at mid-block sections". Journal of the Eastern Asia Society for Transportation Studies, 6: 119-126.
[4] Chiguma, M. L. M. (2007). "Analysis of Side Friction Impacts on Urban Road Links: case study, Dar-es-Salaam”, Doctoral Thesis, Royal Institute of Technology, Stockholm, Sweden.

[5] Le, X., Lu, J. J, Mierzejewski, E. A. and Zhou, Y. (2000). "Variations in Capacity at Signalized Intersections with Different Area Types". Transportation Research Record 1710, Paper No 00-1664, pp 199-204.

[6] Bonneson, J., Nevers, B., Zeeger, J., Nguyen, T. and Fong, F. (2005). "Guidelines for Quantifying the Influence of Area Type and other Factors on Saturation Flow Rates", Texas Transportation Research Institute, Project No. PR385-V2, June, 2005.

[7] Obiri-Yeboah, A. A. (2015). Development of Area-Type Adjustment Factors for Saturation Flow Estimation at Signalized Intersections in Kumasi. Doctoral Thesis, College of Engineering, Kwame Nkrumah University of Science and Technology, Kumasi, Ghana.

[8] Tarko, A. and Tracz, M. (2000). Uncertainty in Saturation Flow Predictions, Proceedings of Fourth International Symposium on Highway Safety, June 27-July 1, Maui, Hawaii, published as Transportation Research Circular E-C018, Transportation Research Board, National Research Council, Washington, D. C., pp. 310-321.

[9] Obiri-Yeboah, A. A., Tuffour, Y. A. and Salifu, M. (2014). "Passenger Car Equivalents at Signalised Intersections within the Kumasi Metropolis in Ghana". IOSR Journal of Engineering, 4 (4): 24-29.

[10] Tuffour, Y. A., Obiri-Yeboah, A. A., Salifu, M. and Acquah, P. C. (2014). "Adjusting for the Effect of Bus Blockage on Saturation Flow Rates". International Journal of Science and Research (IJSR), 3 (6): 749-753. 